\title{
VENTAJAS DE LA PERFORACIÓN DIRECCIONAL: REVISIÓN Y APLICACIÓN EN EL ORIENTE ECUATORIANO.
}

Dario Cedeño, Jenny Fuentes, Carlos Portilla, Carlos Malavé, Johnny Villao 


\title{
Ventajas de la perforación direccional: revisión y aplicación en el Oriente Ecuatoriano \\ Advantages of directional drilling: review and application in the East of Ecuador
}

\author{
Cedeño Macías, Darío ${ }^{(1)}$ Fuentes Cuasquer, Jenny ${ }^{(2)}$ \\ Portilla Lazo, Carlos ${ }^{(1)}$ Malavé Carrera, Carlos ${ }^{(1)}$ \\ Villao Borbor, Jonny ${ }^{(1)}$ \\ Universidad Estatal Península de Santa Elena (UPSE) \\ Campus La Libertad, vía principal Santa Elena - La Libertad \\ La Libertad - Ecuador \\ dcedeno@upse.edu.ec \\ Universidad Industrial de Santander (UIS) \\ Campus Bucaramanga, Carrera 27 Calle 9 \\ Santander - Colombia
}

\begin{abstract}
Resumen
El avance evolutivo que ha tenido la industria hidrocarburífera a nivel mundial, específicamente en las operaciones de extracción y producción, requieren métodos más precisos y rigurosos para llevar a cabo su cometido, considerando principalmente la relación costo beneficio. Para ello es imperativo desarrollar tecnologías y sistemas de manera paralela a dicha evolución. El objetivo de este trabajo es presentar una revisión actualizada de los aspectos esenciales de la perforación direccional, con énfasis en sus evidentes ventajas respecto a la perforación vertical o convencional, y sobre todo su impacto en la reducción de costos para la industria petrolera. De manera que el nivel académico trabaje estrechamente con el nivel profesional, teniendo como parte medular el análisis de datos de ingeniería de diseño en los sistemas de perforación direccional rotativos y su impacto en la reducción de costos operativos en el proceso de extracción de hidrocarburos. Se presentan y discuten los resultados del desempeño alcanzado por estas herramientas en el campo.
\end{abstract}

Palabras Claves: Perforación Direccional, Sistemas de rotación continua, Geonavegación, Perforación Horizontal

\begin{abstract}
The evolutionary advance of the hydrocarbon industry worldwide, specifically in the extraction and production operations, requires more precise and rigorous methods to carry out its mission, considering mainly the cost benefit relation. To do this, it is imperative to develop technologies and systems in parallel with this evolution. The objective of this work is to present an updated review of the essential aspects of directional drilling, with emphasis on its obvious advantages over vertical or conventional drilling, and especially its impact on reducing costs for the oil industry. So that the academic level works closely with the professional level, having as a core part the analysis of design engineering data in rotary directional drilling systems and its impact on the reduction of operating costs in the hydrocarbon extraction process. The results of the performance achieved by these tools in the Auca field are presented and discussed.
\end{abstract}

Keywords: Directional Drilling, Rotary Steerable System, Geosteering, Horizontal Drilling. 


\section{Introducción}

A inicios del siglo XX la explotación de hidrocarburos en Ecuador se llevó a cabo con una gran intensidad, por lo tanto los yacimientos fáciles y someros disminuyeron rápidamente, volviendo la perforación una actividad estratégica, ya que cada vez son mayores las profundidades que se debe alcanzar, las ubicaciones y condiciones son más severas, y es por eso que esta actividad debió apoyarse en distintas disciplinas para obtener el éxito deseado y descubrir nuevas reservas. Una de esas disciplinas es el desplazamiento de la trayectoria de un pozo sobre la horizontalidad; en otras palabras, hacer una perforación direccional.

Es conocido que la distancia más corta entre dos puntos es una línea recta. Sin embargo cuando se trata de realizar extracción de hidrocarburos de subsuelo, este principio no es el más adecuado, pudiendo no ser la vía más rápida ni la más económica. En vista de ello la industria de extracción de hidrocarburos opta frecuentemente por trayectorias de pozos complejas para alcanzar objetivos distantes pero totalmente redituables, aunque para ello dichas trayectorias deban atravesar muchas complicaciones geológicas tales como: fracturas, bloques con múltiples fallas, domos salinos o simplemente alcanzar mayores profundidades en el yacimiento.

Aunque generalmente este tipo de perfil es más difícil de perforar, aumenta de manera significativa la eficiencia de drenaje ya que es mayor la exposición del pozo perforado a la zona productora. Por ende las trayectorias horizontales con altos grados de complejidad son de momento la culminación de la evolución de la perforación direccional.

\section{Evolución de la tecnología de la perforación direccional}

La desviación intencional controlada de los pozos de petróleo se incorporó en la práctica a fines de la década de 1920 cuando se requería evitar obstrucciones, perforar pozos de alivio y evitar determinadas locaciones en las cuales se encontraban construcciones civiles; de hecho esta técnica se empleó incluso para evitar desviación en pozos verticales. [1].

De cierto modo, la destreza para perforar pozos desviados surgió del desarrollo de la perforación rotatoria y de las brocas tricónicas. El diseño de estas brocas permitió que se desplacen lateralmente, en respuesta a diversos parámetros de la formación y de la perforación, tales como el buzamiento y dureza de la formación, la velocidad de rotación ("revolutions per minute", RPM) el peso sobre la broca ("weight on bit", WOB) y el diseño del cono de la misma. Los ingenieros de perforación experimentados reconocieron la tendencia natural de la broca de avanzar hacia la derecha de manera un tanto predecible. Sabiendo esto se intentaba establecer recurrentemente cierto ángulo de avance para compensar con anticipación la deriva entre la ubicación en superficie y el objetivo en el fondo del pozo (figura \#1).

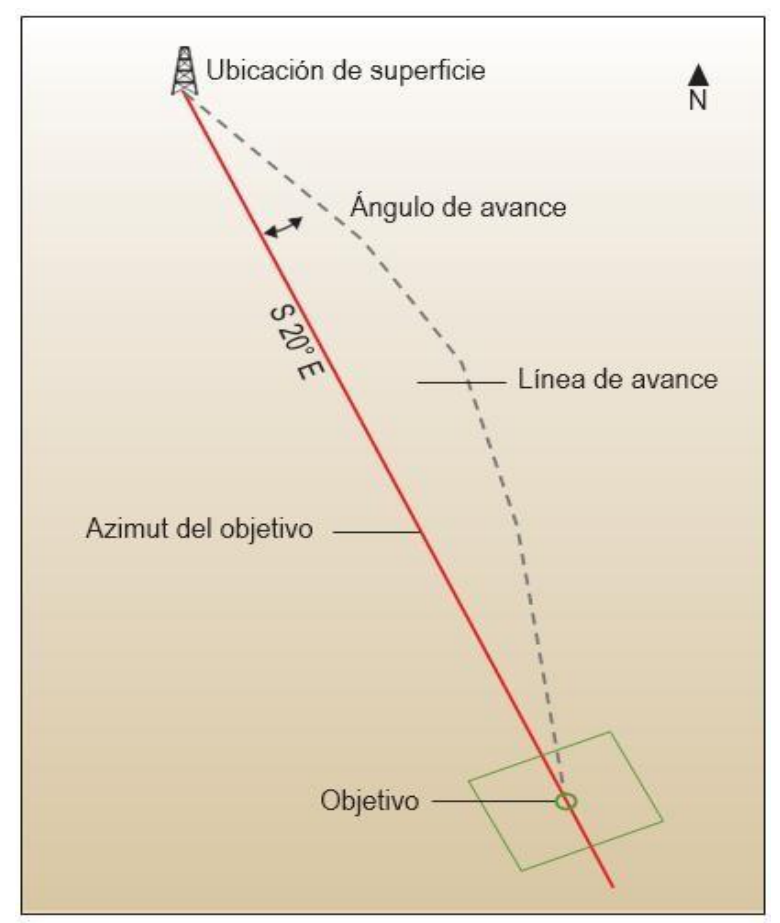

Figura \#1. Ángulo de avance, vista en planta.

Los ingenieros de perforación también identificaron que, mediante alteraciones al conjunto de fondo de pozo ( "bottom hole assembly", BHA) se podría variar el ángulo de inclinación respecto a la trayectoria. Mediante dicha variación en la posición del estabilizador, se podía afectar el equilibrio del BHA, llevándolo a aumentar, mantener o disminuir la inclinación del pozo con respecto a la vertical, comúnmente conocidos como crecimiento angular, retención angular o declinación angular, respectivamente. [1].

La tasa a la cual un BHA construye o hace caer el ángulo se ve afectada por variables tales como la distancia entre estabilizadores, el diámetro y la rigidez del collar de perforación, el buzamiento de la formación, las RPM, peso sobre la broca, la dureza de la formación y el tipo de broca. Tiempo después se adicionó un motor direccional, para realizar la direccionalidad del pozo tanto en azimut e inclinación. (Figura \# 2) 


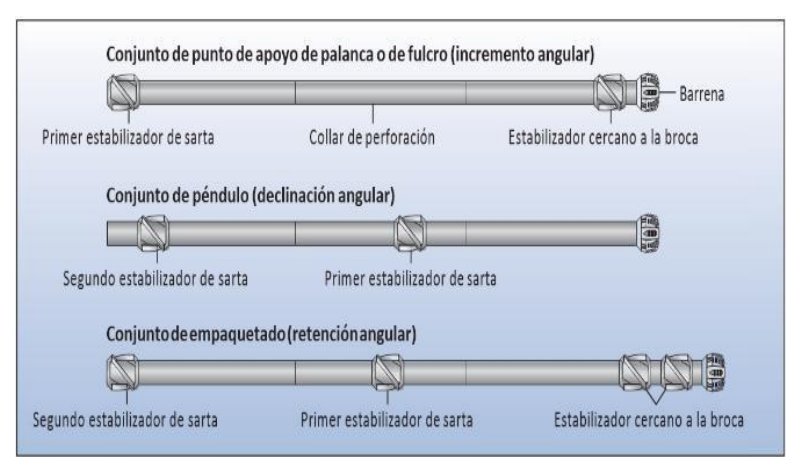

Figura \#2. Configuraciones de BHAs.

Cortesía: Precision Drilling

Actualmente el uso de motores de fondo que usan el flujo de lodo para producir rotación se lo conoce como método convencional para perforar de forma direccional un pozo, el cual junto con una carcasa angular orientan la cara del motor a la dirección requerida. Mientras el motor está en modalidad de deslizamiento (sliding), el resto de la sarta de perforación se encuentra estática, generando un aumento considerable en los parámetros de torque y arrastre a lo largo de la sarta de perforación resultando en fluctuaciones de la orientación, peso irregular en la broca y elevados índice de tortuosidad. Estas condiciones podrían causar problemas posteriores cuando se instala la tubería de revestimiento y se completa el pozo. [2]

Estos parámetros aumentan su criticidad cuando la complejidad direccional se incrementa al igual que la longitud de la sección horizontal. (Figura \# 3)

\section{Ventajas de la tecnología direccional rotatoria}

Considerando los factores anteriormente detallados los especialistas en perforación direccional evolucionaron el diseño de la sarta de perforación, desarrollando así herramientas direccionales rotatorias las cuales son capaces de realizar cambios ya sea en la

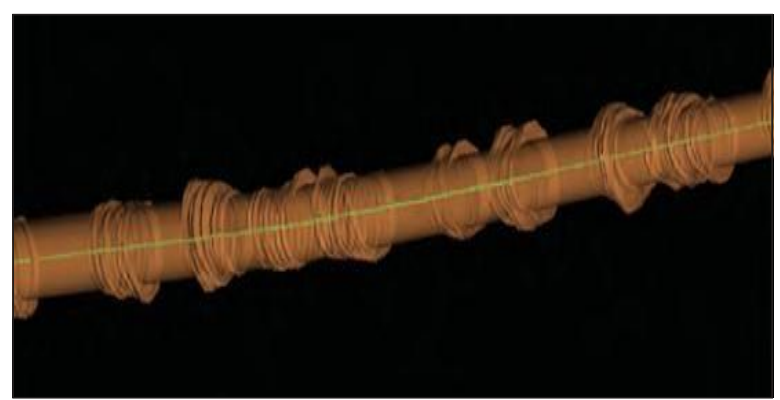

Figura \#3. Análisis de calidad de un pozo. El registro de calibre muestra cómo un motor direccional convencional creó un pozo en forma de espiral.

Cortesía: Schlumberger Limited inclinación o el rumbo mientras toda la sarta de perforación rota continuamente. Estas herramientas producen un hoyo con mayor índice de limpieza, menor rugosidad en las paredes, todo esto mientras reduce el arrastre, mejorando así la transferencia de peso a la broca e incrementando la tasa de penetración. (Figura \# 4)

Los sistemas direccionales rotatorios constituyen una tecnología única y confiable para optimizar todas las aplicaciones de perforación direccional, desde realizar un ("kick off point", KOP) vertical en hoyos de 18 1/4" o 17 1/2", hasta secciones tangenciales en hoyos de 12 $1 / 4$ ", pozos de alcance extendido en secciones de $81 / 2$ " 0 inclusive en hoyos de 5 3/4". Para aplicaciones en secciones de hoyo superficiales, los sistemas de rotación continua mejoran significativamente el rendimiento y minimiza los problemas asociados con la instalación de la tubería de revestimiento, así como también problemas que pueden ser causados, por el torque excesivo de perforación, permitiendo un mejor drenaje del reservorio en la perforación de pozos en 3 dimensiones de alcance extendido. [3,4]

En otras palabras los sistemas de perforación direccional de rotación continua son:

Perforación dentro de objetivos pequeños. Los sistemas ("Rotary Steerable System", RSS) permiten alcanzar con gran precisión objetivos pequeños con un factor de recobro menor a 1 millón de barriles, los cuales serían muy difíciles de alcanzar con la tecnología convencional.

Secciones tangenciales largas. Proporcionan mayor control direccional, secciones con menor tortuosidad, y corridas más largas. Mientras que la tecnología convencional genera muchas veces secciones tangencial donde se alternan las modalidades de rotar y deslizar, provocando elevadas tasas de ruidos mecánicos.

Geonavegación compleja en el reservorio en combinación con sistemas ("Loggin While Drilling", LWD). Permite un control direccional más rápido y más preciso para poner el pozo en el lugar adecuado.

Sidetrack en hoyo abierto. Los sistemas RSS permiten ahorrar significativamente tiempo y dinero eliminando viajes, la necesidad de tapones de cemento y corridas de motor de fondo para desviar la trayectoria cuando se realiza una desviación lateral (Sidetrack).

Desviación desde la verticalidad. Las herramientas pueden realizar desviaciones desde la verticalidad y orientadas a cualquier dirección, sin necesidad de realizar dos viajes. Proceso que no lo pueden hacer herramientas con magnetómetros convencionales. 
Diseño de pozos de bajo ángulo. En el pasado un pozo de 3000 metros verticales que requería un desplazamiento de 300 metros al objetivo era de geometría "S" generalmente, donde se hacia el punto de desvío (KOP) para levantar hasta 30 grados y luego hacer caer el ángulo cuando los 300 metros de desplazamiento eran alcanzados. Con el sistema RSS, el diseño del pozo puede ser hecho para levantar hasta 6 grados por ejemplo, y así mantener la inclinación hasta la profundidad total, y así reduce radicalmente el ángulo máximo, el acumulamiento de recorte, severidades de patas de perro ("Dog Leg Severity", DLS), así como también el alto torque y la fatiga de tubería. Por lo tanto, este tipo de pozos pueden ser perforados más rápidamente corriendo la tubería de revestimiento de manera más fácil. Contrariamente, las sartas navegables con motores de fondo son muy difíciles de controlar a un ángulo bajo y se pierde mucho tiempo deslizando. [5]

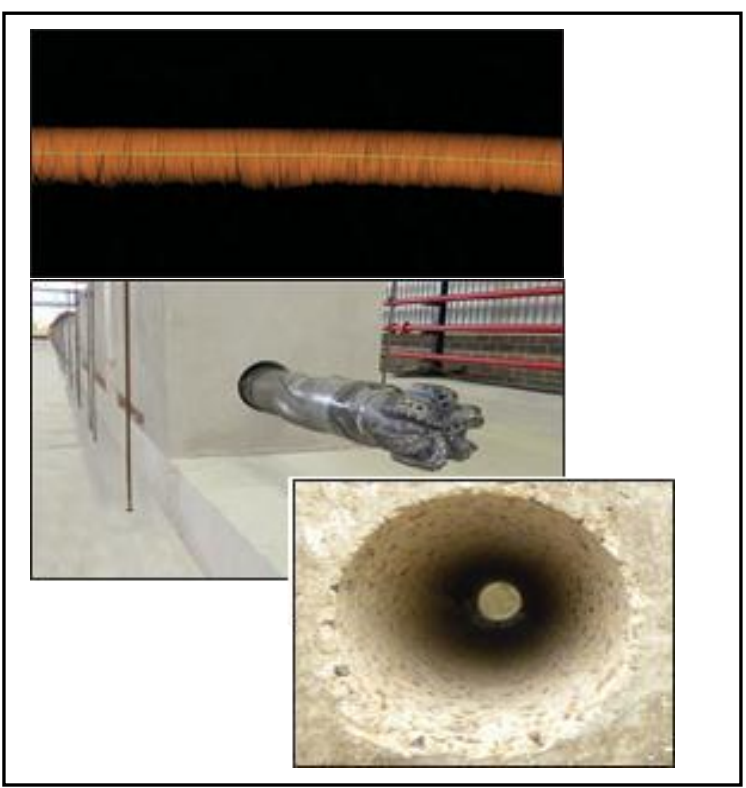

Figura \#4. Análisis de calidad de un pozo. El registro muestra que tanto las paredes del pozo en campo, como los bloques de prueba en laboratorio quedan finalmente con altos porcentajes de uniformidad.

Cortesía: Schlumberger Limited

\section{Funcionamiento del sistema de rotación continua direccional}

Las herramientas de rotación continua son un sistema compacto y poco complicado desde el punto de vista mecánico, ya que incorpora una sección rígida y una unidad de control que añaden únicamente de 3 a $4 \mathrm{~m}$ a la longitud total del BHA. La sección rígida, ubicada directamente detrás de la broca, aplica fuerza sobre la broca en una dirección controlada mientras toda la columna gira. La unidad de control, que se encuentra detrás de la unidad rígida, contiene dispositivos electrónicos, sensores (acelerómetros, inclinómetros magnetómetros, y giróscopos) como mecanismos de control que proporcionan la magnitud y la dirección promedio de las cargas del lado de la mecha, necesarias para alcanzar la trayectoria deseada.

La unidad rígida contiene tres almohadillas retráctiles, las cuales trabajan aplicando el principio "empujando la broca (push the bit)", es decir posicionan la broca de perforación hacia la dirección establecida en el desarrollo del diseño del pozo, las almohadillas se extienden de manera dinámica desde un alojamiento giratorio para crear una fuerza lateral dirigida contra la formación, la cual a su vez, provoca un cambio en la dirección de la perforación mientras rota a la misma velocidad de la tubería. Estas almohadillas son activadas por el flujo de lodo controlado a través de una válvula. Dicha válvula utiliza la diferencia de presión de lodo existente entre el interior y el exterior de la unidad rígida, accionando las almohadillas dirigiendo el lodo en forma sucesiva a la cámara del pistón de cada almohadilla, a medida que rota para alinearse con el punto de empuje deseado en el pozo, que es el punto opuesto a la trayectoria deseada. Una vez que una almohadilla pasa el punto donde aplica la fuerza contraria, la válvula corta el suministro de lodo y el mismo se escapa a través de una compuerta especialmente diseñada para la filtración del lodo. Cada almohadilla se extiende no más de $1 \mathrm{~cm}$ [3/8 pulgada] durante cada vuelta de la unidad rígida. Un eje conecta la válvula con la unidad de control para regular la posición del punto de empuje. [6]

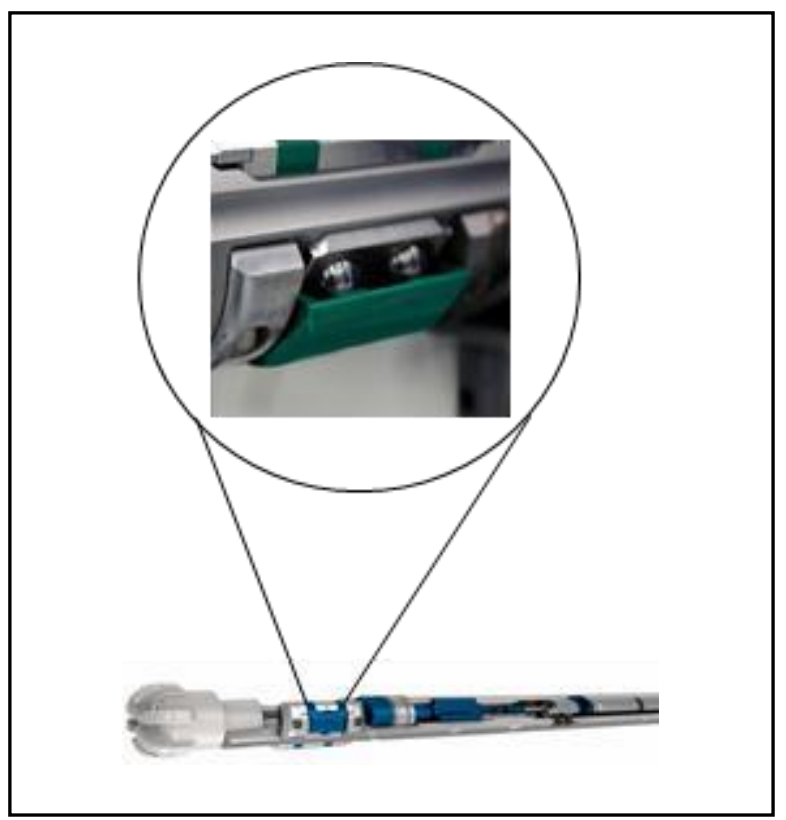

Figura \# 5. Almohadillas laterales de la Unidad Desviadora del sistema RSS, utilizando el principio "Push the bit". Cortesía: Schlumberger Limited 
Si el ángulo del eje se encuentra geoestacionario con respecto a la roca, la broca de perforación será empujada constantemente en una dirección, que es la dirección opuesta al punto de empuje. (Figura \# 5)

\section{Conceptos básicos}

La destreza de controlar la trayectoria del pozo no es suficiente para garantizar en su totalidad la construcción de un pozo perfecto, para que la perforación direccional resulte exitosa es imperativo realizar una cuidadosa planificación de dicha trayectoria.

Para ello se requiere que, geólogos, geofísicos e ingenieros de perforación trabajen de manera colectiva desde el inicio del proyecto de perforación. Una vez determinada la ubicación en superficie y un objetivo deseado en el subsuelo (reservorio), el departamento de ingeniería de planificación direccional debe evaluar los costos, la exactitud requerida y los factores técnicos y geológicos requeridos para obtener el perfil apropiado del hueco (oblicuo, en forma de $\mathrm{S}$, horizontal o quizás con una forma mucho más compleja). La perforación dentro de otro hueco, evento llamado colisión, es totalmente inadmisible, por lo cual se utiliza un programa para evitar dicha colisión con el fin de planificar una trayectoria segura. Para ello es necesario el uso de la comunicación en tiempo real mediante sondas de respuesta rápida —un sistema telemétrico de corta distancia que no requiere cableado - acerca de la posición actual del BHA y la posibilidad de evaluar la formación, todo esto mientras se está perforando, gracias al sistema de ("Measured While Drilling”, MWD y LWD. [2,7]

Dicha sonda de respuesta rápida conecta la interfaz del sistema telemétrico con el sistema MWD por medio de pulsos magnéticos y confirma que las instrucciones han sido recibidas en superficie.

Por lo tanto con los sistemas rotativos direccionales la variedad de brocas que se pueden utilizar es mayor que en el caso de los motores direccionales convencionales, puesto que el control de la orientación de la herramienta es suficiente aun utilizando brocas para zonas altamente agresivas, debido a que se generan grandes fluctuaciones en el torque, alterando la orientación de la herramienta en detrimento del control direccional.

Para un mejor entendimiento del lector se formula diagramáticamente el perfil de un pozo direccional en el cual es utilizado el sistema de rotación continua, demostrando así las complejidades a la que son sometidas dichas herramientas. (Figura \# 6)

\section{Resultados Reales}

Hace poco tiempo atrás, en los campos petroleros del oriente ecuatoriano los sistemas direccionales convencionales dominaban los proyectos de perforación con altas tasa de variaciones angulares (DLS). Sin embargo sus capacidades direccionales, requerían el uso de mayor tiempo de equipo de perforación, ya que para perforar una sección vertical era necesario utilizar un BHA rotativo convencional.

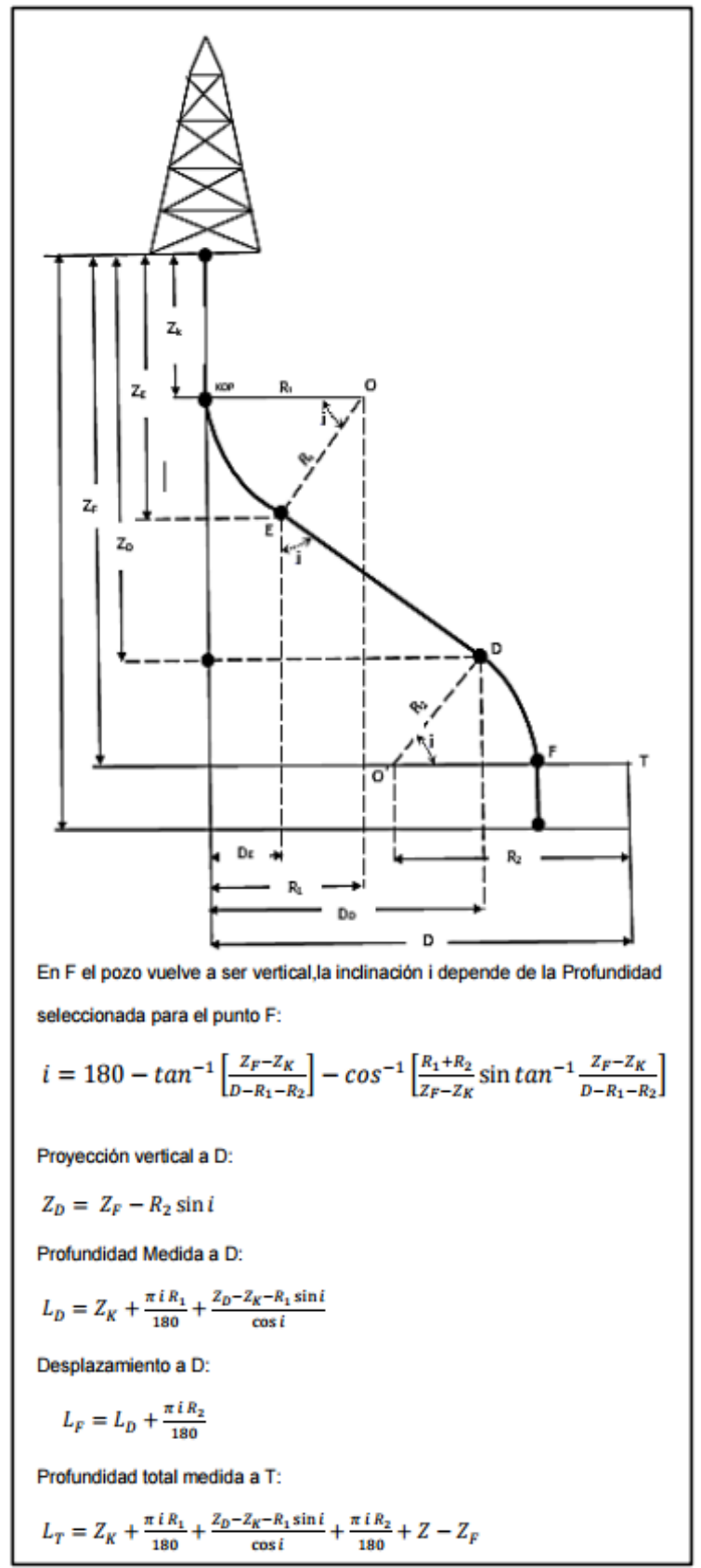

Figura \# 6. Cálculo de los puntos característicos del perfil vertical teórico. Pozo tipo s: $r 1+r 2<D$. 
Al momento de alcanzar el punto de desvío (KOP), el perforador debe extraer totalmente la sarta de perforación desde el punto en subsuelo hasta superficie para cambiarlo por un BHA que integre un motor direccional y continuar la perforación, lo cual se traduce en mayor costo operativo por barril extraído.

La primera vez que se utilizó el sistema RSS, en el Bloque 61 del Oriente Ecuatoriano, fue en el campo Auca. Las secciones de 16" y 12 1/4" fueron perforadas en 4 días cada una, significando un ahorro de 14 días sobre el plan original, lo cual traducido en valor monetario significó un ahorro de cerca de un millón de dólares.

La aplicación de dicho sistema permitió un control direccional y una eficiencia sin precedentes, con una tasa de penetración (ROP) que alcanzó los 300 pies por hora. [8].

Utilizando sensores para monitorear la limpieza del hoyo midiendo la carga anular en tiempo real, y eliminando micro severidades, minimizando arrastres durante los viajes cortos, evitando así el viaje de calibración ya que la geometría del pozo mejoró considerablemente, en relación a los pozos perforados con sistemas convencionales. Y, al no aplicar las modalidades de deslizar y rotar, la perforación con el sistema RSS logró minimizar efectos de tortuosidad del pozo, y elevados índices de torque y arrastre causados por una mala calidad o geometría del pozo, permitiendo perforar secciones laterales mucho más extendidas aumentando el factor de recobro del yacimiento. [9, $10]$.

Uno de los mayores retos evidentemente fueron las intercalaciones de arcillas expancivas las cuales tienen una estructura cristalina de tres capas (una capa de alúmina y dos capas de sílice) y que presentan la característica común de hinchamiento o expansión de su red mineralógica por hidratación cuando se exponen al agua-componente principal de los fluidos utilizados en la perforación- aumentando su volumen.

Durante el proceso se utilizó fluido de perforación compuesto por el sistema PHPA (Poliacrilamida parcialmente hidrolizada), con altos contenidos de sales como el cloruro de sodio $(\mathrm{NaCl})$ y el cloruro de potasio $(\mathrm{KCl})$, para generar un intercambio catiónico reduciendo la absorción de agua en las arcillas, dando estabilidad a las mismas y por ende al hoyo. [10]

Ante esto los componentes internos del sistema RSS no tuvieron que ser modificados ya que están diseñados para trabajar con cualquier tipo de fluido de perforación sin afectar su desempeño.

\section{Conclusiones}

Los avances en la tecnología de perforación direccional están ayudando significativamente a la industria de extracción de hidrocarburos que no podían producirse de otra forma. La última generación de sistemas direccionales de rotación continua está alcanzando trayectorias de pozo que eran inimaginables anteriormente, al mismo tiempo que disminuyen los costos y los riesgos en los pozos mejorando su producción. Estas trayectorias de pozo cada vez más complejas están estimulando a la industria a avanzar más en la búsqueda de nuevas reservas.

Ecuador no es un caso aislado ya que cada vez es menos probable encontrar nuevas reservas hidrocarburíferas, por lo tanto es imperativo la utilización de tecnología de punta y especializada que permita aumentar el factor de recobro de las reservas que actualmente están siendo explotadas.

\section{Recomendaciones}

- Incluir en las operaciones de perforación de pozos horizontales con sistemas de rotación continua en campos ecuatorianos, herramientas de geonavegación azimutal que establezcan los límites de la formación productora de manera precisa para obtener un factor de recobro cerca del $90 \%$.

- Aumentar la tasa de circulación un 25\% arriba de la tasa promedio que se manejó en la perforación, esto permitirá que el BHA con sistema RSS no se quede atrapado en la secciones con radios de curvaturas elevados debido a la rigidez de mismo.

- Seleccionar brocas de diamante compacto policristalino (PDC), con mayor cantidad de cortadores en los hombros, al momento de atravesar formaciones volcánicas para ayudar a la direccionalidad del pozo.

\section{Agradecimientos}

Especial agradecimiento a: Schlumberger Limited, Weatherford International, Halliburton, y Precision Drilling, The Society of Petroleum Engineers. 


\section{Bibliografía}

[1] Perneder, L.; Detournay, E. Equilibrium Inclinations of Straight Boreholes. University of Minnesota. 160335-PA SPE Journal Paper - 2013.

[2] Akinniranye, G., Megat, A., Elsweisy, H., Palacio, J., Poedjono, B., \& Goobie, R. B. Implementation of a Shock and Vibration Mitigation Process: Achieving Real-Time Solutions and Savings. Society of Petroleum Engineers. doi: 10.2118/107903-PA. 2009

[3] Figueredo, C. Enhanced RSS Technology Pushes Drilling Envelope. Society of Petroleum Engineers. doi: 10.2118/03140032-JPT. 2014

[4] Zimmer, C., Richter, D., Person, J., Tilley, J., \& Bittar, M. Drilling a Better Pair: New Technologies in SAGD Directional Drilling. Society of Petroleum Engineers. doi: 10.2118/137137-PA. 2012.

[5] Sugiura, J., Bowler, A., \& Lowdon, R. Improved Continuous Azimuth and Inclination Measurement by Use of a RotarySteerable System Enhances DownholeSteering Automation and Kickoff Capabilities Near Vertical. Society of Petroleum Engineers. doi: 10.2118/166099-PA. 2014.

[6] Wilson, A. Bending Rules With High-BuildRate Rotary-Steerable Systems. Society of Petroleum Engineers. doi:10.2118/12160062-JPT.2016
[7] Carrizo, H. R., Sanchez, J. L., Perdomo, L., \& Bertarelli, M. Changing the Way To Drill in Ecuador: Experience Using Point-the-Bit and Hybrid RSS in Horizontal and 3D Complex Wells. Society of Petroleum Engineers. doi:10.2118/169295-MS. 2014

[8] Barton, S. P., Clarke, A. J., Garcia, A., Perez, D., Mora, G., \& Carrion, C. Improved Drilling Performance: Downhole Dynamic Logging Tools Break Paradigm in Ecuador. Society of Petroleum Engineers. doi:10.2118/122208MS. 2009

[9] Sierra, F., Teran, N., Bastidas, M., Soria, G., Rojas, E., Villalva, R., Castro, B. Horizontal Well Landing Optimization Combining Rotary Steerable Technology and Deep Azimuthal Measurements in Block 7, Ecuador. Society of Petroleum Engineers. doi:10.2118/177147-MS. 2015

[10] Manohar Lal. Shale Stability: Drilling Fluid Interaction and Shale Strength. SPE Latin American and Caribbean Petroleum Engineering Conference. 1999 